Check for updates

For numbered affiliations see end of article.

Correspondence to: L Azoulay laurent.azoulay@mcgill.ca (or @LaurentAzoulay0 on Twitter ORCID 0000-0001-5162-3556)

Additional material is published online only. To view please visit the journal online.

Cite this as: $B M J$ 2018;363:k4209 http://dx.doi.org/10.1136/bmi.k4209

Accepted: 17 September 2018

\title{
Angiotensin converting enzyme inhibitors and risk of lung cancer: population based cohort study
}

\author{
Blánaid M Hicks, ${ }_{1,2,3}$ Kristian B Filion, ${ }^{1,2,4}$ Hui Yin, ${ }^{1}$ Lama Sakr, ${ }^{5}$ Jacob A Udell,,${ }^{6,7}$ \\ Laurent Azoulay ${ }^{1,2,8}$
}

\section{ABSTRACT}

OBJECTIVE

To determine whether the use of angiotensin converting enzyme inhibitors (ACEIs), compared with use of angiotensin receptor blockers, is associated with an increased risk of lung cancer.

DESIGN

Population based cohort study.

SETTING

United Kingdom Clinical Practice Research Datalink.

\section{PARTICIPANTS}

A cohort of 992061 patients newly treated with antihypertensive drugs between 1 January 1995 and 31 December 2015 was identified and followed until 31 December 2016.

\section{MAIN OUTCOME MEASURES}

Cox proportional hazards models were used to estimate adjusted hazard ratios with $95 \%$ confidence intervals of incident lung cancer associated with the time varying use of ACEls, compared with use of angiotensin receptor blockers, overall, by cumulative duration of use, and by time since initiation.

RESULTS

The cohort was followed for a mean of 6.4 (SD 4.7) years, generating 7952 incident lung cancer events (crude incidence 1.3 (95\% confidence interval 1.2 to 1.3) per 1000 person years). Overall, use of ACEls was associated with an increased risk of lung cancer (incidence rate $1.6 \vee 1.2$ per 1000 person years; hazard ratio $1.14,95 \%$ confidence interval 1.01 to 1.29), compared with use of angiotensin receptor blockers. Hazard ratios gradually increased with longer durations of use, with an association evident after five years of use (hazard ratio $1.22,1.06$ to 1.40 )

\section{WHAT IS ALREADY KNOWN ON THIS TOPIC}

Biological evidence suggests that angiotensin converting enzyme inhibitors may increase the risk of lung cancer through the accumulation of bradykinin and substance $P$ in the lung

However, observational studies examining this association are limited and report inconsistent results

\section{WHAT THIS STUDY ADDS}

The use of angiotensin converting enzyme inhibitors was associated with a $14 \%$ increased risk of lung cancer

Associations were evident after five years of use and increased with longer durations of use, particularly in patients who used angiotensin converting enzyme inhibitors for more than 10 years

The magnitudes of the observed estimates are modest, but these small relative effects could translate into large absolute numbers of patients at risk, so these findings should be replicated in other settings and peaking after more than 10 years of use (1.31, 1.08 to 1.59 ). Similar findings were observed with time since initiation.

\section{CONCLUSIONS}

In this population based cohort study, the use of ACEls was associated with an increased risk of lung cancer. The association was particularly elevated among people using ACEls for more than five years. Additional studies, with long term follow-up, are needed to investigate the effects of these drugs on incidence of lung cancer.

\section{Introduction}

Angiotensin converting enzyme inhibitors (ACEIs) are effective drugs used in the treatment of hypertension. ${ }^{1}$ Although these drugs have been shown to be relatively safe in the short term, concerns have been raised that their long term use may be associated with an increased risk of cancer. These concerns have been subject to debate, with observational studies producing mixed findings, ${ }^{2-4}$ including with respect to lung cancer. ${ }^{24}$ Some biological evidence exists for a possible association between ACEIs and risk of lung cancer. The use of ACEIs causes an accumulation of bradykinin in the lung, ${ }^{5}$ which has been reported to stimulate growth of lung cancer. ${ }^{56}$ ACEI use also results in accumulation of substance $\mathrm{P}$, which is expressed in lung cancer tissue and has been associated with tumor proliferation and angiogenesis. $^{7}$

Meta-analyses of randomized controlled trials found no evidence of an increase in cancer incidence with ACEIs, but most had relatively small sample sizes and short durations of follow-up (median 3.5 years). ${ }^{89}$ The few observational studies that have investigated the association between ACEI use and lung cancer have reported mixed findings. ${ }^{10-17}$ However, most of these studies were designed to assess the risk of cancer overall and not lung cancer specifically. ${ }^{10-16}$ Additionally, several of these studies had some methodological shortcomings, including short duration of followup (for example, median of 0.7 years), ${ }^{17}$ failure to account for cancer latency, ${ }^{12} 131517$ and immortal time bias. ${ }^{15}$ Furthermore, results of some studies may have been influenced by the use of an inappropriate comparator group, introducing potential confounding by indication, ${ }^{14}$ and the inclusion of prevalent users of antihypertensives. ${ }^{15}$

Thus, in light of the conflicting and limited evidence from both preclinical and observational studies, we conducted a large, population based study to determine whether the use of ACEIs, compared with use of angiotensin receptor blockers, is associated with an increased risk of lung cancer. 


\section{Methods}

Data source

This study used the UK Clinical Practice Research Datalink (CPRD). The CPRD includes data from approximately 700 general practices comprising more than 15 million patients; these have been shown to be representative of the UK population. ${ }^{18}$ The CPRD records demographic information, anthropometric data (such as body mass index), lifestyle information (such as smoking status and alcohol use), medical diagnoses and procedures (coded using the Read code classification ${ }^{19}$ ), and prescription data (coded according to the UK Prescription Pricing Authority's dictionary ${ }^{20}$ ), which have been shown to be valid and of high quality. ${ }^{21} 22$ Furthermore, lung cancer diagnoses recorded in the CPRD have been shown to be highly concordant $(>93 \%)$ with those recorded in the UK National Cancer Data Repository. ${ }^{23}$

\section{Study population}

We identified a base cohort of all patients, at least 18 years of age, who were newly treated with an antihypertensive drug (including $\beta$ adrenoceptor blockers, $\alpha$ adrenoceptor blockers, ACEIs, angiotensin receptor blockers, calcium channel blockers, vasodilators, centrally acting antihypertensives, diuretics, ganglion blockers, and renin inhibitors) between 1 January 1988 and 31 December 2015. We required all patients to have at least one year of medical history in the CPRD before their first ever prescription for an antihypertensive drug. This was necessary to ensure the inclusion of new users of antihypertensive drugs, thus minimizing the possibility of prevalent user bias. $^{24}$

From the base cohort defined above, we identified a study cohort of all patients who started taking a new antihypertensive drug class on or after 1 January 1995 (the first year in which both ACEIs and angiotensin receptor blockers were available in the UK) until 31 December 2015. These patients included those newly treated with an antihypertensive drug class (that is, first ever antihypertensive prescriptions) as well as those who added on or switched to an antihypertensive drug class not previously used in their treatment history. We defined cohort entry as the date of this first prescription. We excluded patients with a previous diagnosis of any cancer (other than nonmelanoma skin cancer) and those who had received treatments for cancer (chemotherapy or radiotherapy) at any time before cohort entry. This was to ensure the identification of incident cases of lung cancer during follow-up and to avoid the inclusion of patients with metastatic lesions to the lung from other cancer sites. Finally, we excluded patients with less than one year of follow-up after cohort entry for latency considerations and to ensure the identification of incident events during follow-up.

We followed up all patients who met the study inclusion criteria, starting one year after cohort entry, until a diagnosis of incident lung cancer (identified on the basis of Read codes; supplementary table A) or censoring on death from any cause, end of registration with the general practice, or the end of the study period (31 December 2016), whichever occurred first.

\section{Exposure assessment}

We used a time varying exposure definition in which each person day of follow-up was classified into one of three mutually exclusive exposure categories: ACEIs (alone or in combination with other antihypertensive drugs, but no previous use of angiotensin receptor blockers), angiotensin receptor blockers (alone or in combination with other non-ACEI antihypertensive drugs), and other antihypertensive drugs. The latter category also included patients who switched from an ACEI to an angiotensin receptor blocker and vice versa; these may represent an atypical group in which switching may have been motivated by side effects, such as cough in the case of ACEIs, which may in turn lead to increased detection of lung cancer. ${ }^{24}$ We introduced a one year exposure lag period to account for a minimum latency time window and to minimize reverse causality. Thus, we considered patients starting an antihypertensive drug to be unexposed until one year after the date of the first prescription and exposed thereafter. The reference category was angiotensin receptor blockers, as these drugs are recommended at the same disease stage, thereby minimizing potential confounding by indication. ${ }^{25}$

\section{Potential confounders}

All models were adjusted for the following variables measured at cohort entry: age, sex, year of cohort entry, body mass index (modeled as a continuous variable by using a restricted cubic spline with five interior knots), smoking status (current, former, never), alcohol related disorders (including alcoholism, alcoholic cirrhosis of the liver, alcoholic hepatitis, and hepatic failure), and history of lung diseases (including pneumonia, tuberculosis, and chronic obstructive pulmonary disease), all measured at any time before cohort entry. In addition, models included duration of treated hypertension (defined as the time between first ever prescription for an antihypertensive drug and cohort entry) and use of statins at any time before cohort entry. Finally, the models were adjusted for the total number of unique drug classes prescribed in the year before cohort entry, as a general measure of comorbidity. ${ }^{26}$

\section{Statistical analyses}

We calculated crude incidence rates of lung cancer and $95 \%$ confidence intervals, based on the Poisson distribution, for each exposure group. We used time dependent Cox proportional hazards models to estimate hazard ratios and $95 \%$ confidence intervals of lung cancer associated with the use of ACEIs compared with the use of angiotensin receptor blockers, using multiple imputation for variables with missing values. ${ }^{27}{ }^{28}$ We used ordinal logistic regression and linear regression models to impute variables with missing information (for smoking and body mass 
index, respectively) with explanatory variables and cumulative hazard (as recommended, ${ }^{29}$ and ACEI use at cohort entry), along with all confounders mentioned previously. We did five imputations and combined the results by using Rubin's rules. ${ }^{30}$

\section{Secondary analyses}

We did three secondary analyses. Firstly, we assessed whether a duration-response relation existed between cumulative duration of ACEI use and incidence of lung cancer. For this time dependent analysis, we estimated hazard ratios in a time dependent manner for three predefined duration categories: five years or less, 5.1-10 years, and longer than 10 years. Secondly, we investigated the association between time since starting ACEIs and risk of lung cancer, estimating hazard ratios for three predefined categories: five years or less, 5.1-10 years, and longer than 10 years. We also modeled cumulative duration of use and time since initiation as continuous variables, using restricted cubic spline models with five knots to produce a smooth curve of the hazard ratio as a function of duration. ${ }^{3132}$ To investigate possible effect modification by smoking status, we included an interaction term between the exposure and smoking status variables. Additionally, we repeated the primary and secondary analyses among non-smokers.

\section{Sensitivity analyses}

We did three sensitivity analyses to assess the robustness of our findings. Firstly, given uncertainties related to the length of the latency time window, we varied the length of the exposure lag period to two and three years. Secondly, as an alternate means of controlling for confounding, we repeated the analysis by stratifying the model on tenths of disease risk score (supplementary methods 1). ${ }^{33}$ Finally, we repeated the analysis using a marginal structural Cox proportional hazards model using inverse probability of treatment and censoring weighting-a method designed to adjust for time dependent confounding associated with time varying exposures (supplementary methods 2). ${ }^{3536}$

\section{Ancillary analyses}

We did two ancillary analyses to consider the possibility that angiotensin receptor blockers may be associated with a decreased risk of lung cancer incidence. $^{37}$ The first compared ACEIs with thiazide diuretics, as the latter have not been previously associated with lung cancer incidence. For this analysis, we redefined exposure hierarchically into four mutually exclusive categories: ACEIs (alone or in combination with other antihypertensive drugs, but no previous use of thiazide diuretics or angiotensin receptor blockers), thiazide diuretics (alone or in combination with other non-ACEI or non-angiotensin receptor blocker antihypertensive drugs), angiotensin receptor blockers (alone or in combination with other non-ACEI or non-thiazide antihypertensive drugs), and other antihypertensive drugs. The second analysis compared angiotensin receptor blockers with thiazide diuretics to assess whether the former are associated with a decreased risk of lung cancer. For this analysis, we redefined exposure hierarchically as angiotensin receptor blockers (alone or in combination with other antihypertensive drugs, but no previous use of thiazide diuretics or ACEIs), thiazide diuretics (alone or in combination with other non-ACEI or non-angiotensin receptor blocker antihypertensive drugs), ACEIs (alone or in combination with other non-angiotensin receptor blocker or non-thiazide antihypertensive drugs), and other antihypertensive drugs. For both analyses, we assessed the association overall and by cumulative duration of use. We used SAS version 9.4 and $\mathrm{R}$ for all analyses.

\section{Patient and public involvement}

Our study was a secondary data analysis and did not include patients as study participants. No patients were involved in setting the research question or the outcome measures, nor were they involved in the design and implementation of the study. There are no plans to involve patients in the dissemination of results, nor will we disseminate results directly to patients, beyond our general media communications plan.

\section{Results}

The cohort included 992061 patients (fig 1) followed for a mean of 6.4 (SD 4.7) years beyond the one year post-cohort entry latency period. During the followup period, 335135 patients were treated with ACEIs, 29008 with angiotensin receptor blockers, and 101637 with both ACEIs and angiotensin receptor blockers. The three most commonly used ACEIs were ramipril (26\%; 257420 patients) lisinopril (12\%; 120641 patients), and perindopril (7\%; 70955 patients). Overall, 7952 patients were newly diagnosed as having lung cancer during 6350584 person years of follow-up, generating a crude incidence rate of 1.3 (95\% confidence interval 1.2 to 1.3$)$ per 1000 person years.

Table 1 shows baseline characteristics of the entire cohort and by use of ACEIs, angiotensin receptor blockers, and other antihypertensive drugs at cohort entry. Compared with angiotensin receptor blocker users, ACEI users were more likely to be male, to have alcohol related disorders, to be current smokers, and to have a higher body mass index. Additionally, ACEI users had a shorter duration of treated hypertension and were more likely to have used statins and other prescription drugs. ACEI and angiotensin receptor blocker users had a similar history of pneumonia, tuberculosis, and chronic obstructive pulmonary disease.

Table 2 shows the results from primary and secondary analyses. Compared with angiotensin receptor blockers, ACEIs were associated with an overall $14 \%$ greater risk of lung cancer $(1.6 v 1.2$ per 1000 person years; hazard ratio $1.14,95 \%$ confidence interval 1.01 to 1.29$)$. In secondary analyses, the use of ACEIs for less than five years was not associated 


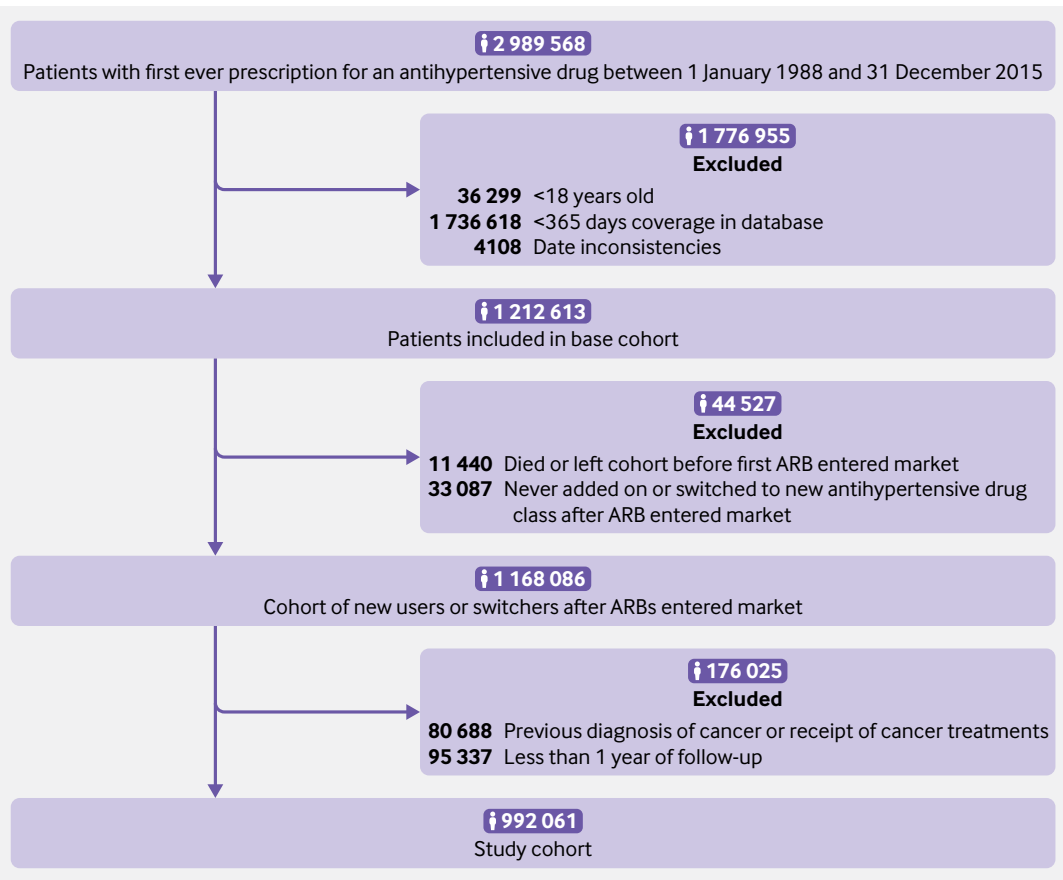

Fig 1 | Study flow diagram describing construction of base cohort and study cohort. $A R B=$ angiotensin receptor blocker ratios increasing with longer times since initiation, peaking at more than 10 years since initiation (hazard ratio $1.29,1.10$ to 1.51 ). We saw similar patterns in analyses using restricted cubic splines (supplementary figures A and B). Smoking status did not significantly modify the association between ACEI use and risk of lung cancer ( $\mathrm{P}$ for interaction $=0.40$; supplementary table B). Supplementary table $\mathrm{C}$ shows analyses conducted within non-smokers. Overall, the results were consistent with those of the primary analyses, with the hazard ratio increasing with longer cumulative durations of use ( $>10$ years cumulative use: hazard ratio $1.64,1.02$ to 2.64 ).

\section{Sensitivity analyses}

Results of sensitivity analyses are summarized in figure 2 and supplementary tables D-G. Overall, these yielded consistent results, generating hazard ratios ranging between 1.13 and 1.22. The latter estimate was from the marginal structural model that controlled for potential time dependent confounding.

\section{Ancillary analyses}

Compared with the use of thiazide diuretics, the use of ACEIs was associated with a 6\% increased risk of lung cancer (hazard ratio 1.06, 1.00 to 1.13) (supplementary table H). Similar to the main analysis, use of ACEIs for less than five years was not associated with an increased risk of lung cancer, whereas hazard ratios were elevated with increasing use, peaking with more than 10 years of use $(1.23,1.04$ to 1.44$)$. Analysis comparing angiotensin receptor blockers with thiazide with an increased risk of lung cancer (hazard ratio $1.10,0.96$ to 1.25$)$. However, the hazard ratio was elevated with five to 10 years of use $(1.22,1.06$ to 1.40) and continued to increase with more than 10 years of use $(1.31,1.08$ to 1.59$)$. Similar associations were observed for time since starting ACEI, with hazard

\begin{tabular}{|c|c|c|c|c|}
\hline \multirow[b]{2}{*}{ Characteristic } & \multirow[b]{2}{*}{ Entire cohort } & \multicolumn{3}{|c|}{ Antihypertensive drug use at cohort entry } \\
\hline & & ACEls & ARBs & Others \\
\hline Total & 992061 & $208353(21.0)$ & $16027(1.6)$ & $767681(77.4)$ \\
\hline Mean (SD) age, years & $55.6(16.6)$ & $57.8(13.1)$ & $57.9(13.2)$ & $54.9(17.5)$ \\
\hline Male sex & $459064(46.3)$ & $133091(63.9)$ & $9591(59.8)$ & $316382(41.2)$ \\
\hline Alcohol related disorders & $71605(7.2)$ & $18199(8.7)$ & $1092(6.8)$ & $52314(6.8)$ \\
\hline \multicolumn{5}{|l|}{ Smoking status: } \\
\hline Current & $215098(21.7)$ & $41595(20.0)$ & $2802(17.5)$ & $170701(22.2)$ \\
\hline Past & $227504(22.9)$ & $58683(28.2)$ & $3916(24.4)$ & $164905(21.5)$ \\
\hline Never & $484831(48.9)$ & $99820(47.9)$ & $8248(51.5)$ & $376763(49.1)$ \\
\hline Unknown & $64628(6.5)$ & $8255(4.0)$ & $1061(6.6)$ & $55312(7.2)$ \\
\hline \multicolumn{5}{|l|}{ Body mass index: } \\
\hline$<25$ & $303311(30.6)$ & $45164(21.7)$ & $3602(22.5)$ & $254545(33.2)$ \\
\hline $25-30$ & $304699(30.7)$ & $71655(34.4)$ & $5447(34.0)$ & $227597(29.6)$ \\
\hline$\geq 30.0$ & $224888(22.7)$ & $67353(32.3)$ & $4724(29.5)$ & $152811(19.9)$ \\
\hline Unknown & $159163(16.0)$ & $24181(11.6)$ & $2254(14.1)$ & $132728(17.3)$ \\
\hline Mean (SD) duration of treated hypertension, years & $0.2(1.5)$ & $0.3(1.8)$ & $0.5(2.4)$ & $0.2(1.4)$ \\
\hline Pneumonia & $22403(2.3)$ & $5027(2.4)$ & $320(2.0)$ & $17056(2.2)$ \\
\hline Tuberculosis & $2399(0.2)$ & $474(0.2)$ & $37(0.2)$ & $1888(0.2)$ \\
\hline Chronic obstructive pulmonary disease & $78669(7.9)$ & $16152(7.8)$ & $1180(7.4)$ & $61337(8.0)$ \\
\hline Statins & $164891(16.6)$ & $73510(35.3)$ & $4092(25.5)$ & $87289(11.4)$ \\
\hline Mean (SD) total No of unique drug classes & $4.1(4.1)$ & $4.1(4.1)$ & $3.8(4.1)$ & $4.2(4.1)$ \\
\hline 0 & $150293(15.2)$ & $35384(17.0)$ & 3107 (19.4) & $111802(14.6)$ \\
\hline 1 & 147609 (14.9) & 31022 (14.9) & $2603(16.2)$ & $113984(14.8)$ \\
\hline 2 & $135085(13.6)$ & $27027(13.0)$ & $2195(13.7)$ & $105863(13.8)$ \\
\hline 3 & $115121(11.6)$ & $22157(10.6)$ & $1740(10.9)$ & $91224(11.9)$ \\
\hline$\geq 4$ & $443953(44.8)$ & $92763(44.5)$ & $6382(39.8)$ & $344808(44.9)$ \\
\hline
\end{tabular}




\begin{tabular}{|c|c|c|c|c|c|c|}
\hline \multirow[b]{2}{*}{ Exposure* } & \multirow[b]{2}{*}{ Events } & \multirow[b]{2}{*}{ Person years } & \multirow[b]{2}{*}{ Incidence rate $(95 \% \mathrm{Cl}) \dagger$} & \multicolumn{2}{|c|}{ Hazard ratio $(95 \% \mathrm{Cl})$} & \multirow[b]{2}{*}{ P for trend } \\
\hline & & & & Crude & Adjusted $¥$ & \\
\hline ARBs & 266 & 213557 & $1.2(1.1$ to 1.4$)$ & 1.00 & 1.00 (reference) & \\
\hline ACEIS & 3186 & 1977139 & $1.6(1.6$ to 1.7$)$ & 1.32 & 1.14 (1.01 to 1.29$)$ & \\
\hline \multicolumn{7}{|c|}{ Cumulative duration of ACEI use (years) } \\
\hline$\leq 5$ & 2084 & 1440232 & $1.4(1.4$ to 1.5$)$ & 1.24 & $1.10(0.96$ to 1.25$)$ & \multirow{3}{*}{$<0.001$} \\
\hline $5.1-10$ & 905 & 457309 & $2.0(1.9$ to 2.1$)$ & 1.44 & $1.22(1.06$ to 1.40$)$ & \\
\hline$>10$ & 197 & 79598 & $2.5(2.1$ to 2.8$)$ & 1.63 & 1.31 (1.08 to 1.59$)$ & \\
\hline \multicolumn{7}{|c|}{ Time since first ACEI use (years) } \\
\hline$\leq 5$ & 1617 & 1158441 & $1.4(1.3$ to 1.5$)$ & 1.24 & 1.11 (0.97 to 1.27$)$ & \multirow{3}{*}{$<0.001$} \\
\hline $5.1-10$ & 1155 & 647103 & $1.8(1.7$ to 1.9$)$ & 1.33 & $1.14(0.99$ to 1.30$)$ & \\
\hline$>10$ & 414 & 171596 & $2.4(2.2$ to 2.7$)$ & 1.62 & $1.29(1.10$ to 1.51$)$ & \\
\hline
\end{tabular}

$\mathrm{ACEl}=$ angiotensin converting enzyme inhibitor; $\mathrm{ARB}=$ angiotensin receptor blocker.

*Use of other antihypertensive drugs (including of use of both ACEIs and ARBs) was considered in model but not shown in table; these generated 4500 lung cancer events and 4159887 person years.

tPer 1000 person years.

₹Adjusted for age, sex, year of cohort entry, body mass index, smoking, alcohol related disorders, history of lung diseases before cohort entry (including pneumonia, tuberculosis, and chronic obstructive pulmonary disease), duration of treated hypertension, use of statins, and total number of unique drug classes in year before cohort entry.

diuretics showed null associations overall (hazard ratio $0.93,0.82$ to 1.06 ) and by cumulative duration of use (supplementary table I).

\section{Discussion}

In this large population based study of nearly one million patients, the use of ACEIs was associated with an overall $14 \%$ increased risk of lung cancer. Associations were evident after five years of use and increased with longer durations of use, particularly among patients who used ACEIs for more than 10 years (31\% increased risk). Although the magnitudes of the observed associations are modest, ACEIs are one of the most widely prescribed drug classes; in the UK, 70.1 million antihypertensives are dispensed each year, of which approximately $32 \%$ are ACEIs. ${ }^{38} 39$ Thus, small relative effects could translate into large absolute numbers of patients at risk for lung cancer. Given the potential impact of our findings, they need to be replicated in other settings, particularly among patients exposed for longer durations.

\section{Comparison with previous studies}

Although meta-analyses of randomized controlled trials found no evidence of an association between the use of ACEIs and cancer overall, or lung cancer specifically, ${ }^{89}$ these trials were not powered or designed to assess these outcomes. Moreover, with relatively

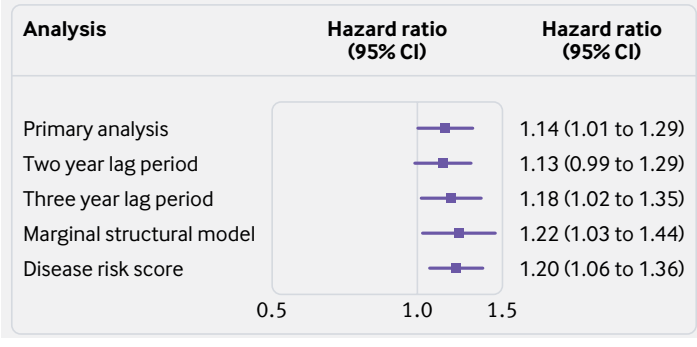

Fig 2 | Forest plot summarizing results of primary and sensitivity analyses assessing association between angiotensin converting enzyme inhibitor use and lung cancer incidence short durations of follow-up (median duration of 3.5 (range 1.3-5.1) years), these trials did not have sufficient follow-up to assess long term adverse events such as cancer. ${ }^{89}$ This is particularly important given that an association between use of ACEIs and risk of lung cancer became evident after five years of use in our study. To our knowledge, although several observational studies reported on the association between ACEIs and lung cancer incidence, ${ }^{10-16}$ only one study was specifically designed to investigate this association. ${ }^{17}$ In this well conducted study, the use of ACEIs was not associated with an increased risk of lung cancer (hazard ratio $0.99,0.84$ to 1.16 ), compared with angiotensin receptor blockers. However, as this study had a maximum follow-up of five years, its conclusion is not incompatible with our finding suggesting no association in the first five years of use (hazard ratio $1.10,0.96$ to 1.25). Other observational studies have investigated this association, but their findings were part of secondary analyses and thus should be interpreted with caution. Overall, these studies produced mixed results, with some reporting increased risks, ${ }^{1011} 16$ others reporting null associations, ${ }^{12-14}$ and one study reporting a $66 \%$ decreased risk. ${ }^{15}$ However, the latter study may have been affected by immortal time bias, which resulted from the misclassification of unexposed person time as exposed person time. ${ }^{40}$ The other studies had other limitations, such as the inclusion of prevalent users of antihypertensive drugs, ${ }^{15}$ confounding by indication, ${ }^{14}$ and not accounting for cancer latency in their analyses. ${ }^{12} 131517$

The association between ACEIs and lung cancer is biologically plausible. In addition to angiotensin I, angiotensin converting enzyme also metabolizes bradykinin, an active vasodilator. ${ }^{41}$ Thus, the use of ACEIs results in the accumulation of bradykinin in the lung. ${ }^{5}$ Bradykinin receptors have been located on various cancerous tissues including lung cancer, ${ }^{5} 42$ and bradykinin may directly stimulate growth of lung cancer. ${ }^{5}{ }^{6}$ Bradykinin has been shown to stimulate the release of vascular endothelial growth factor, thus promoting angiogenesis, ${ }^{43} 44$ as well as 
having indirect effects on lung cancer by enhancing vascular permeability, via the activation of matrix metalloproteinase, facilitating tumor invasion and metastases. $^{44}$ Moreover, ACEI use also results in accumulation of substance $\mathrm{P}$, which is expressed in lung cancer tissue and is associated with tumor

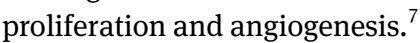

The results of this study also raise important questions about the new angiotensin receptor/ neprilysin inhibitor sacubitril/valsartan. Neprilysin inhibition results in increases in vasoactive and other peptides including bradykinin and substance $\mathrm{P}^{45}$ The recent PARADIGM-HF trial reported clinical benefits for cardiovascular outcomes and death; however, cancer events were not reported. ${ }^{46}$ Therefore, whether these new renin-angiotensin system inhibitors may also increase the risk of lung cancer in the long term remains unknown. Moreover, these results also raise questions about recent evidence suggesting that ACEIs may protect against radiation induced pneumonitis in patients with lung cancer. ${ }^{47}$ Although limited studies have suggested improvements in survival in patients with lung cancer receiving renin-angiotensin system inhibitors and tyrosine kinase inhibitors or chemotherapy, the effect of ACEIs specifically on lung cancer progression remains uncertain. ${ }^{48} 49$

\section{Strengths and limitations of study}

This study has several strengths. Firstly, to our knowledge, with more than 990000 patients followed for an average of 6.4 years (beyond the one year postcohort entry lag period), this is the largest study to have been conducted to specifically assess this association. Secondly, we used a new-user design, thus minimizing biases related to the inclusion of prevalent users. ${ }^{50}$ Thirdly, we used a time varying exposure definition that eliminated immortal time bias, while also accounting for cancer latency. Finally, the use of the CPRD allowed us to adjust the models for several potential important confounders, including smoking status, which was not available in some of the previous studies. ${ }^{17}$

This study has some limitations. Firstly, although we were able to adjust for several important confounders, this study lacked information on other potential confounders such as socioeconomic status, diet, exposure to radon or asbestos, and family history of lung cancer. ${ }^{51} 52$ Additionally, despite adjusting for smoking status, we lacked detailed information on duration and intensity of smoking, which have been shown to be associated with lung cancer incidence. ${ }^{5152}$ However, an analysis conducted within non-smokers produced results consistent with those of the primary analyses, with a clear duration-response association, providing reassurance that residual confounding by smoking did not materially affect our findings. Secondly, prescriptions in the CPRD represent those written by general practitioners, so misclassification of exposure is possible if patients did not adhere to the treatment regimen or received prescriptions from specialists. However, as all patients entering the cohort were those newly treated with antihypertensive drugs, misclassification due to non-adherence should be minimal and likely non-differential between ACEI and angiotensin receptor blocker users.

Thirdly, we compared ACEIs with angiotensin receptor blockers, as the latter also act on the reninangiotensin system and are used at the same disease stage but have not been associated with neuropeptide accumulation in the lung. However, angiotensin receptor blockers may also have an effect on lung cancer incidence, ${ }^{53}$ and a meta-analysis of observational studies reported a decreased risk with these drugs. ${ }^{37}$ Studies included in this meta-analysis had some limitations, and several compared angiotensin receptor blockers with ACEIs. ${ }^{37}$ Thus, the apparent protective effect of angiotensin receptor blockers may be the result of a deleterious effect of ACEIs on lung cancer incidence. ${ }^{37}$ Nevertheless, our study was designed to consider this possibility by comparing ACEIs with thiazide diuretics in ancillary analyses. Reassuringly, this analysis yielded consistent results, both in terms of overall association and by cumulative duration of use. Importantly, our analyses comparing angiotensin receptor blockers with thiazide diuretics produced null associations for both overall and cumulative duration of use; this suggests that the observed increased risk with ACEIs is unlikely to be attributable to the purported antitumor effects of angiotensin receptor blockers. Fourthly, misclassification of the outcome is possible; however, lung cancer has been shown to be well recorded in the CPRD when compared with the UK National Cancer Data Repository (concordance rate of $93 \%) .{ }^{23}$ Associations may also vary by subtypes of lung cancer, but this information was not available within the CPRD.

Finally, persistent cough is a common and well known side effect of ACEIs, raising the possibility that the observed association could be due to detection bias. Patients taking ACEIs may be more likely to undergo diagnostic evaluations, such as computerized tomography of the chest, leading to an increased detection of preclinical lung cancers. Information on chest investigations is not well recorded in the CPRD, so we could not account for this possibility in our analyses. However, a recent study found minimal evidence of differences in chest investigations after ACEI and angiotensin receptor blocker initiation. ${ }^{17}$ Moreover, an over-detection of lung cancer would be expected to be observed relatively soon after the start of treatment, which is one the reasons why our exposures were lagged by one year. Lengthening the exposure lag period to two and three years yielded similar findings to those observed for the primary analysis. Furthermore, associations between ACEI use and lung cancer risk were evident only with increasing durations of use (after at least five years of use). Taken together, these results do not corroborate the hypothesis of an overdetection of lung cancer among ACEIs

\section{Conclusions}

In this large, population based study, the use of ACEIs was associated with an elevated risk of lung cancer 
overall, along with evidence of a duration-response relation. Although the magnitudes of the observed estimates are modest, these small relative effects could translate into large absolute numbers of patients at risk for lung cancer, so these findings need to be replicated in other settings.

\section{AUTHOR AFFILIATIONS}

${ }^{1}$ Centre for Clinical Epidemiology, Lady Davis Institute, Jewish General Hospital, Montreal, QC, Canada, H3T 1E2 ${ }^{2}$ Department of Epidemiology, Biostatistics and Occupational Health, McGill University, Montreal, QC, Canada ${ }^{3}$ Centre for Public Health, School of Medicine, Dentistry and Biomedical Sciences, Queen's University Belfast, Belfast, UK ${ }^{4}$ Division of Clinical Epidemiology, Department of Medicine, McGill University, Montreal, QC, Canada

${ }^{5}$ Division of Pulmonary Diseases, Department of Medicine, Jewish General Hospital, Montreal, QC, Canada

${ }^{6}$ Women's College Research Institute and Cardiovascular Division, Department of Medicine, Women's College Hospital, University of Toronto, Toronto, ON, Canada

${ }^{7}$ Cardiovascular Division, Department of Medicine, Peter Munk Cardiac Centre, Toronto General Hospital, University of Toronto, Toronto, ON, Canada

${ }^{8}$ Gerald Bronfman Department of Oncology, McGill University, Montreal, QC, Canada

Contributors: All authors conceived and designed the study, analyzed and interpreted the data, and critically revised the manuscript for important intellectual content. LA acquired the data. BMH, $\mathrm{HY}$, and LA did the analyses. BMH wrote the manuscript, and all authors participated in the interpretation of the results and critical revision of the manuscript. The corresponding author attests that all listed authors meet authorship criteria and that no others meeting the criteria have been omitted. LA is the guarantor.

Funding: This study was funded by a Foundation Scheme grant from the Canadian Institutes of Health Research. BMH holds a Cancer Research UK population research fellowship. KBF holds a chercheur-boursier junior 2 award from the Fonds de recherche du Québec - Santé (FRQS) and is the recipient of a William Dawson scholar award from McGill University. JAU holds a Heart and Stroke Foundation of Canada national new investigator/Ontario clinician scientist (phase I) award. LA holds a chercheur-boursier senior award from the FRQS and is the recipient of a William Dawson scholar award from McGill University. The funding source had no influence on the design and conduct of the study; collection, management, analysis, and interpretation of the data; preparation, review, or approval of the manuscript; or the decision to submit the manuscript for publication.

Competing interests: All authors have completed the ICMJE uniform disclosure form at www.icmje.org/coi_disclosure.pdf (available on request from the corresponding author) and declare: this study was funded by the Canadian Institutes of Health Research; JAU has participated in research projects funded by AstraZeneca, Janssen, Novartis, and Sanofi and received personal consultancy fees and honorariums for symposia from Amgen, AstraZeneca, Boehringer-Ingelheim, Janssen, Merck, Novartis, and Sanofi; these have no relation to the work presented in this manuscript; no other relationships or activities that could appear to have influenced the submitted work.

Ethical approval: The study protocol was approved by the Independent Scientific Advisory Committee of the Clinical Practice Research Datalink (protocol number 16_255R) and by the Research Ethics Board of Jewish General Hospital, Montreal, Quebec, Canada.

Data sharing: No additional data available.

Transparency: The guarantor affirms that this manuscript is an honest, accurate, and transparent account of the study being reported; that no important aspects of the study have been omitted; and that any discrepancies from the study as planned (and, if relevant, registered) have been explained.

This is an Open Access article distributed in accordance with the Creative Commons Attribution Non Commercial (CC BY-NC 4.0) license, which permits others to distribute, remix, adapt, build upon this work non-commercially, and license their derivative works on different terms, provided the original work is properly cited and the use is non-commercial. See: http://creativecommons.org/licenses/ by-nc/4.0/.
1 Heran BS, Wong MM, Heran IK, Wright JM. Blood pressure lowering efficacy of angiotensin converting enzyme (ACE) inhibitors for primary hypertension. Cochrane Database Syst Rev 2008;4:CD003823.

2 Yoon C, Yang HS, Jeon I, Chang Y, Park SM. Use of angiotensinconverting-enzyme inhibitors or angiotensin-receptor blockers and cancer risk: a meta-analysis of observational studies. CMAI 2011;183:E1073-84. doi:10.1503/cmaj.101497

3 Rosenthal T, Gavras I. Angiotensin inhibition and malignancies: a review. J Hum Hypertens 2009;23:623-35. doi:10.1038/jhh.2009.21

4 Shen J, Huang Y-M, Wang M, et al. Renin-angiotensin system blockade for the risk of cancer and death. / Renin Angiotensin Aldosterone Syst 2016;17:1470320316656679. doi:10.1177/1470320316656679

5 Trifilieff A, Da Silva A, Gies JP. Kinins and respiratory tract diseases. Eur Respir/ 1993;6:576-87.

6 Sethi T, Rozengurt E. Multiple neuropeptides stimulate clonal growth of small cell lung cancer: effects of bradykinin, vasopressin, cholecystokinin, galanin, and neurotensin. Cancer Res 1991;51:3621-3.

7 Muñoz M, Coveñas R. Involvement of substance P and the NK-1 receptor in human pathology. Amino Acids 2014:46:1727-50. doi:10.1007/s00726-014-1736-9

8 ARB Trialists Collaboration. Effects of telmisartan, irbesartan, valsartan, candesartan, and losartan on cancers in 15 trials enrolling 138,769 individuals. J Hypertens 2011;29:623-35. doi:10.1097/HJH.0b013e328344a7de

9 Bangalore S, Kumar S, Kjeldsen SE, et al. Antihypertensive drugs and risk of cancer: network meta-analyses and trial sequential analyses of 324,168 participants from randomised trials. Lancet Oncol 2011;12:65-82. doi:10.1016/S1470-2045(10)70260-6

10 Azoulay L, Assimes TL, Yin H, Bartels DB, Schiffrin EL, Suissa S. Long-term use of angiotensin receptor blockers and the risk of cancer. PLoS One 2012;7:e50893. doi:10.1371/journal.pone.0050893

11 Bhaskaran K, Douglas I, Evans S, van Staa T, Smeeth L. Angiotensin receptor blockers and risk of cancer: cohort study among people receiving antihypertensive drugs in UK General Practice Research Database. BM/ 2012;344:e2697. doi:10.1136/bmj.e2697

12 Chang C-H, Lin J-W, Wu L-C, Lai M-S. Angiotensin receptor blockade and risk of cancer in type 2 diabetes mellitus: a nationwide case-control study. J Clin Oncol 2011;29:3001-7. doi:10.1200/JCO.2011.35.1908

13 Friis S, Sørensen HT, Mellemkjaer L, et al. Angiotensin-converting enzyme inhibitors and the risk of cancer: a population-based cohort study in Denmark. Cancer 2001;92:2462-70. doi:10.1002/1097-0142(20011101)92:9<2462::AIDCNCR1596>3.0.CO;2-L

14 Hallas J, Christensen R, Andersen M, Friis S, Bjerrum L. Long term use of drugs affecting the renin-angiotensin system and the risk of cancer: a population-based case-control study. Br I Clin Pharmacol 2012:74:180-8, doi:10.1111/j.1365-2125.2012.04170.x

15 Lever AF, Hole DJ, Gillis CR, et al. Do inhibitors of angiotensinI-converting enzyme protect against risk of cancer? Lancet 1998:352:179-84. doi:10.1016/S0140-6736(98)03228-0

16 Pasternak B, Svanström H, Callréus T, Melbye M, Hviid A. Use of angiotensin receptor blockers and the risk of cancer. Circulation 2011:123:1729-36. doi:10.1161 CIRCULATIONAHA. 110.007336

17 Gokhale M, Girman C, Chen Y, Pate V, Funk MJ, Stürmer T. Comparison of diagnostic evaluations for cough among initiators of angiotensin converting enzyme inhibitors and angiotensin receptor blockers. Pharmacoepidemiol Drug Saf 2016;25:512-20. doi:10.1002/pds.3977

18 Herrett E, Gallagher AM, Bhaskaran K, et al. Data Resource Profile: Clinical Practice Research Datalink (CPRD). Int J Epidemiol 2015;44:827-36. doi:10.1093/ije/dyv098

19 National Health Service. Read Codes. 2017. https://digital.nhs.uk/ services/terminology-and-classifications/read-codes

20 NHS Business Services Authority. Dictionary of medicines and devices. 2017. https://www.nhsbsa.nhs.uk/pharmacies-gp-practicesand-appliance-contractors/dictionary-medicines-and-devices-dmd

21 Jick SS, Kaye JA, Vasilakis-Scaramozza C, et al. Validity of the general practice research database. Pharmacotherapy 2003;23:686-9. doi:10.1592/phco.23.5.686.32205

22 Lawrenson R, Williams T, Farmer R. Clinical information for research; the use of general practice databases. J Public Health Med 1999:21:299-304. doi:10.1093/pubmed/21.3.299

23 Boggon R, van Staa TP, Chapman M, Gallagher AM, Hammad TA, Richards MA. Cancer recording and mortality in the General Practice Research Database and linked cancer registries. Pharmacoepidemiol Drug Saf 2013;22:168-75. doi:10.1002/pds.3374

24 Ray WA. Evaluating medication effects outside of clinical trials new-user designs. Am J Epidemiol 2003;158:915-20. doi:10.1093/aje/kwg231 
25 National Institute for Health and Care Excellence. Hypertension in adults: diagnosis and management. 2016. https://www.nice.org.uk/ guidance/cg127/chapter/1-Guidance\#initiating-and-monitoringantihypertensive-drug-treatment-including-blood-pressure-targets-2

26 Schneeweiss S, Seeger JD, Maclure M, Wang PS, Avorn J, Glynn RJ. Performance of comorbidity scores to control for confounding in epidemiologic studies using claims data. Am J Epidemiol 2001;154:854-64. doi:10.1093/aje/154.9.854

27 Schafer LL. Analysis of Incomplete Multivariate Data. Chapman and Hall, 1997. doi:10.1201/9781439821862

28 Rubin DB. Multiple Imputation for Nonresponse in Surveys. John Wiley \& Sons, 1987. doi:10.1002/9780470316696

29 White IR, Royston P. Imputing missing covariate values for the Cox model. Stat Med 2009;28:1982-98. doi:10.1002/sim.3618

30 Sterne JAC, White IR, Carlin JB, et al. Multiple imputation for missing data in epidemiological and clinical research: potential and pitfalls. BMJ 2009;338:b2393. doi:10.1136/bmj.b2393

31 Greenland S. Dose-response and trend analysis in epidemiology: alternatives to categorical analysis. Epidemiology 1995;6:356-65. doi:10.1097/00001648-199507000-00005

32 Heinzl H, Kaider A, Zlabinger G. Assessing interactions of binary time-dependent covariates with time in cox proportional hazards regression models using cubic spline functions. Stat Med 1996;15:2589-601. doi:10.1002/(SICI)10970258(19961215)15:23<2589::AID-SIM373>3.0.CO;2-0

33 Arbogast PG, Ray WA. Performance of disease risk scores, propensity scores, and traditional multivariable outcome regression in the presence of multiple confounders. $\mathrm{Am}$ J Epidemiol 2011;174:613-20. doi:10.1093/aje/kwr143

34 Arbogast PG, Ray WA. Use of disease risk scores in pharmacoepidemiologic studies. Stat Methods Med Res 2009:18:67-80 doi:10.1177/0962280208092347

35 Hernán MA, Brumback B, Robins IM. Marginal structural models to estimate the causal effect of zidovudine on the survival of HIV-positive men. Epidemiology 2000;11:561-70. doi:10.1097/00001648-200009000-00012

36 Robins JM, Hernán MA, Brumback B. Marginal structural models and causal inference in epidemiology. Epidemiology 2000;11:550-60. doi:10.1097/00001648-200009000-00011

37 Zhang J, Liu J, Chen J, et al. Angiotensin receptor blockers (ARBs) reduce the risk of lung cancer: a systematic review and meta-analysis. Int J Clin Exp Med 2015;8:12656-60.

38 Fretheim A, Oxman AD. International variation in prescribing antihypertensive drugs: its extent and possible explanations. BMC Health Serv Res 2005;5:21. doi:10.1186/1472-6963-5-21

39 National Health Service Digital. Prescriptions Dispensed in the Community - Statistics for England, 2004-2014. 2015 https://digital.nhs.uk/catalogue/PUB17644

40 Suissa S. Immortal time bias in pharmaco-epidemiology. Am Epidemiol 2008;167:492-9. doi:10.1093/aje/kwm324
41 Carey RM, Siragy HM. Newly recognized components of the renin-angiotensin system: potential roles in cardiovascular and renal regulation. Endocr Rev 2003;24:261-71. doi:10.1210/er.2003-0001

42 Golias Ch, Charalabopoulos A, Stagikas D, Charalabopoulos K. Batistatou A. The kinin system--bradykinin: biological effects and clinical implications. Multiple role of the kinin system--bradykinin. Hippokratia 2007;11:124-8.

43 Ishihara K, Hayash I, Yamashina S, Majima M. A potential role of bradykinin in angiogenesis and growth of S-180 mouse tumors. Jpn J Pharmacol 2001;87:318-26. doi:10.1254/jjp.87.318

44 Stewart JM. Bradykinin antagonists as anti-cancer agents. Curr Pharm Des 2003;9:2036-42. doi:10.2174/1381612033454171

45 Singh JSS, Burrell LM, Cherif M, Squire IB, Clark AL, Lang CC. Sacubitril/valsartan: beyond natriuretic peptides. Heart 2017:103:1569-77. doi:10.1136/heartjnl-2017-311295

46 McMurray JJV, Packer M, Desai AS, et al, PARADIGM-HF Investigators and Committees. Angiotensin-neprilysin inhibition versus enalapril in heart failure. N Engl J Med 2014;371:993-1004. doi:10.1056/ NEJMoa1409077

47 Sun F, Sun H, Zheng X, et al. Angiotensin-converting Enzyme Inhibitors Decrease the Incidence of Radiation-induced Pneumonitis Among Lung Cancer Patients: A Systematic Review and Meta-analysis. J Cancer 2018;9:2123-31. doi:10.7150/jca.24665

48 Miao L, Chen W, Zhou L, Wan H, Gao B, Feng Y. Impact of Angiotensin I-converting Enzyme Inhibitors and Angiotensin II Type-1 Receptor Blockers on Survival of Patients with NSCLC. Sci Rep 2016;6:21359. doi:10.1038/srep21359

49 Wilop S, von Hobe S, Crysandt M, Esser A, Osieka R, Jost E. Impact of angiotensin I converting enzyme inhibitors and angiotensin II type 1 receptor blockers on survival in patients with advanced non-small-cell lung cancer undergoing first-line platinum-based chemotherapy. J Cancer Res Clin Oncol 2009;135:1429-35. doi:10.1007/s00432-009-0587-3

50 Ray WA. Evaluating medication effects outside of clinical trials: new-user designs. Am J Epidemiol 2003;158:915-20. doi:10.1093/aje/kwg231

51 Subramanian I, Govindan R. Lung cancer in never smokers: a review. J Clin Oncol 2007;25:561-70. doi:10.1200/JCO.2006.06.8015

52 Matakidou A, Eisen T, Houlston RS. Systematic review of the relationship between family history and lung cancer risk. Br J Cancer 2005:93:825-33 doi:10.1038/sj.bjc.6602769

53 Sipahi I, Debanne SM, Rowland DY, Simon DI, Fang JC. Angiotensin-receptor blockade and risk of cancer: meta-analysis of randomised controlled trials. Lancet Oncol 2010;11:627-36. doi:10.1016/S1470-2045(10)70106-6

\section{Supplementary materials}

